\title{
Tratamiento del infarto segmentario idiopático de epiplón mayor en el adulto mediante omentectomía laparoscópica
}

\author{
Carolina Perdomo Reyes* ${ }^{\star}$ Daniel González González†
}

\section{Resumen}

Introducción: el infarto segmentario idiopático de epiplón mayor es una excepcional causa de cuadro agudo de abdomen en el adulto.

Caso clínico: se presenta el caso de un adulto joven que se operó de urgencia con diagnóstico de apendicitis aguda y el abordo por vía laparoscópica, comprobándose en la exploración un infarto segmentario de epiplón mayor que se resolvió mediante la omentectomía parcial con buena evolución y alta precoz.

Discusión: si bien el diagnóstico imagenológico de infarto de epiplón mayor es posible dada su baja incidencia y las características de presentación clínica, su diagnóstico habitualmente es intraoperatorio. Su tratamiento es la omentectomía laparoscópica del sector afectado. Con diagnóstico preoperatorio, puede admitirse una conducta expectante, aunque la persistencia de la sintomatología es causa de reingreso y prolongación del tratamiento definitivo.

Palabras clave: Infarto

Laparoscopía

Epiplón

Key words: Infarction

Omentum

Laparoscopy

\section{Introducción}

El infarto segmentario de epiplón mayor es causa poco frecuente de dolor abdominal agudo simulando diversas enfermedades quirúrgicas ${ }^{(1)}$. Se lo puede clasificar en primario o idiopático y secundario a estrangulación, como contenido de hernias o eventraciones, tumores, traumatismos, procesos inflamatorios intraabdominales, torsiones o adherencias ${ }^{(2)}$.

El objetivo de este trabajo es comunicar un caso clínico en paciente adulto joven cuyo diagnóstico fue intraoperatorio y se trató mediante una omentectomía segmentaria laparoscópica.

\section{Caso clínico}

Paciente de sexo masculino, 36 años, sin antecedentes patológicos a destacar. Consultó por dolor centro abdominal de tres días de evolución, aparición brusca, de moderada intensidad, acompañado de un episodio de vómito. No presentó otras alteraciones digestivas ni urinarias. Al examen, paciente longilíneo en el que se constató temperatura axilar de $37,8^{\circ} \mathrm{C}$, lengua saburral y defensa en fosa ilíaca derecha. De la paraclínica se destacó: 13.700 leucocitos $/ \mathrm{mm}^{3}$. Con diagnóstico de apendicitis aguda se operó de urgencia por abordaje videolaparoscópico. En la exploración se constató hemoperitoneo escaso de sangre oscura a predominio de gotera parietocólica derecha, fosa ilíaca derecha y fondo de saco de Douglas, con infarto segmentario derecho en un epiplón mayor bífido (foto 1). Apéndice cecal de características normales. Se procedió a la omentectomía parcial mediante la sección sobre sector pediculado y sano del epiplón. Se extrajo la pieza en bolsa de nailon por el abordaje de flanco izquierdo que debió ampliarse.

\footnotetext{
* Residente de Cirugía General. ORCID https://orcid.org/0000.0002.0786.1167.

† Profesor titular de Clínica Quirúrgica. ORCID https://orcid.org/0000.0003.3916.9201.

Clínica Quirúrgica 3. Facultad de Medicina. Universidad de la República. Hospital Maciel. Montevideo, Uruguay.

Correspondencia: Carolina Perdomo. Correo electrónico: carolinaperdomo09@gmail.com

Cuenta con consentimiento informado del paciente para su publicación.

Los autores declaran no tener conflicto de intereses.

Recibido: 4/10/19

Aprobado: $27 / 4 / 20$
} 


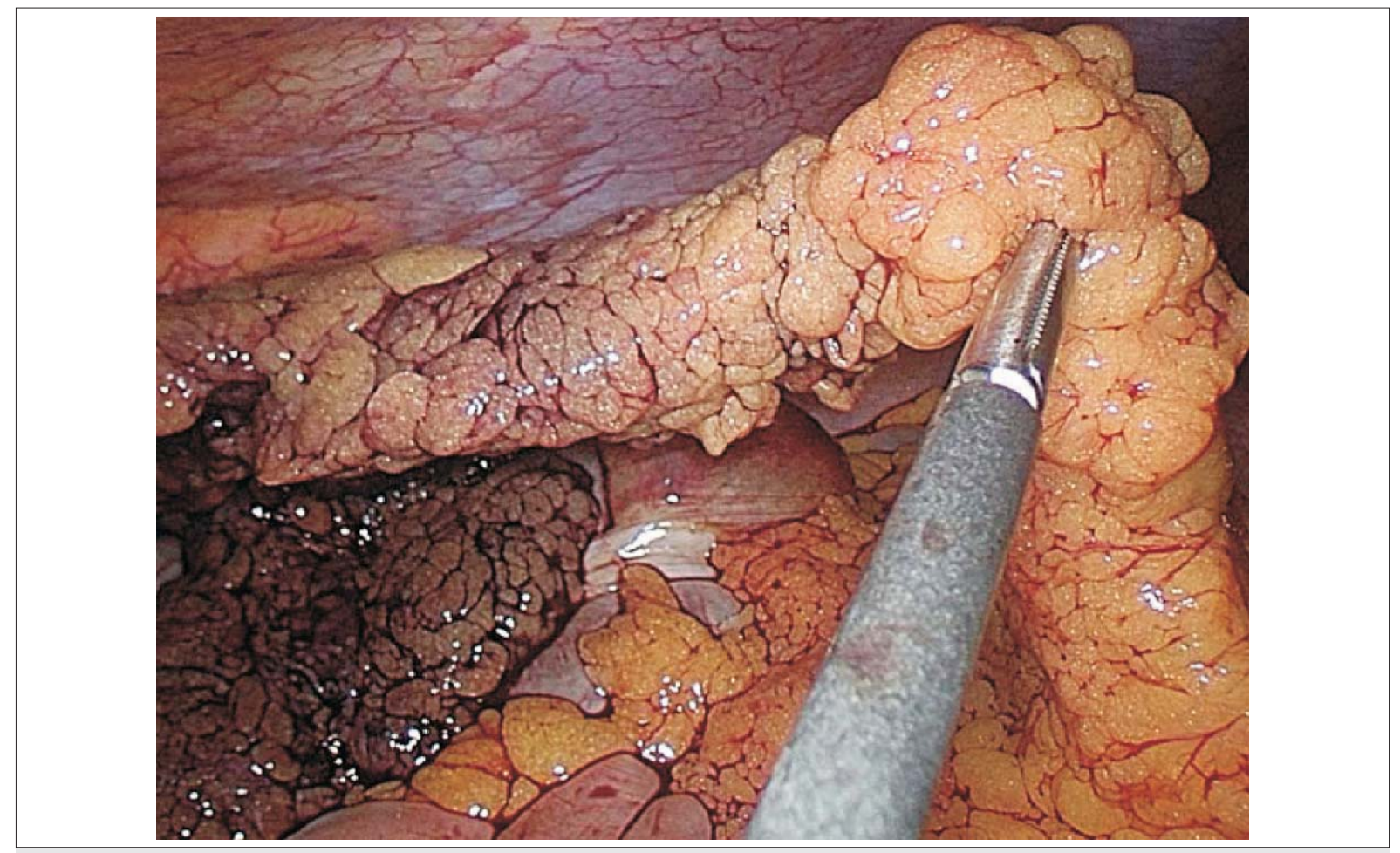

Figura 1. Rama derecha del epiplón mayor bífido. En el sector distal se aprecia el área de infarto y en el proximal, cargado con la pinza, tejido de características normales.

Se completó el procedimiento con la toilette peritoneal. Buena evolución posoperatoria. Alta a las 12 horas.

El resultado de la anatomía patológica confirmó el diagnóstico y no evidenció malignidad.

\section{Discusión}

De acuerdo a los hallazgos clínicos (ausencia de hernias, eventraciones o traumatismos), intraoperatorios (ausencia de procesos inflamatorios, torsiones o adherencias) e informe anatomopatológico (ausencia de procesos tumorales), el caso comunicado puede ser clasificado como un infarto de epiplón primario o idiopático.

Existen aproximadamente 400 casos publicados en la literatura ${ }^{(1)}$. En ellos, predominó en adultos jóvenes y habitualmente obesos, condición considerada como un factor de riesgo no presente en nuestro caso.

Clínicamente se presenta con dolor abdominal de topografía variable dependiendo del sector comprometido, acompañado o no de repercusión digestiva. Particularmente los segmentarios y localizados a derecha simulan una apendicitis aguda y en general es el diagnóstico con el cual llegan a la cirugía ${ }^{(3)}$. Se puede acompañar de aumento leve de parámetros inflamatorios clínicos y paraclínicos, como los presentó nuestro paciente. En niños existe una mayor prevalencia de la afectación a derecha, lo que se vincula a la presencia de un epiplón bífido, va- riación encontrada en el caso presentado, malformaciones en su pedículo vascular y a su mayor movilidad ${ }^{(4)}$.

Tanto la ecografía abdominal como la tomografía computada, en general solicitadas con otros planteos diagnósticos, pueden identificar signos imagenológicos sugestivos y en esos casos es posible una conducta expectante y tratamiento sintomático. Sin embargo, es importante tener presente que en la casuística de McCus$\operatorname{ker}^{(5)}, 22,2 \%$ de los niños en que se decidió dicha consulta reingresaron por dolor.

Como se expresó, la mayoría de los casos se resuelven quirúrgicamente, ya que el diagnóstico preoperatorio en los infartos de topografía derecha es el de apendicitis aguda y es durante la exploración intraoperatoria que se diagnostican ${ }^{(6)}$. El tratamiento es la omentectomía del sector afectado, preservando el epiplón sano.

El abordaje laparoscópico permite realizar el diagnóstico y tratamiento, así como una adecuada exploración de toda la cavidad peritoneal y su toilette ${ }^{(6,7)}$. El abordaje convencional por incisión de Mc Burney, en caso de diagnóstico preoperatorio de apendicitis aguda, limita una correcta exploración, así como el tratamiento, obligando a ampliar la incisión o efectuar una mediana.

Por tanto, si bien la conducta expectante es admitida, la resolución quirúrgica y fundamentalmente por abordaje videolaparoscópico permite una rápida recupera- 
ción, como ocurrió en el caso comunicado, previniendo complicaciones $^{(8)}$.

\section{Summary}

Introduction: idiopathic segmental infarction of the greater omentum constitutes an exceptional cause of an acute condition in adults.

Clinical case: study presents the clinical case of a young adult who underwent an emergency laparoscopic appendectomy for the treamtent of acute apenditicis. The exploration revealed a segmental infarction of the greater omentum that was resolved by partial omentectomy. Evolution was good and the patient was allowed an early discharge.

Discussion: despite the fact an image diagnosis of infarction of the greater omentum is possible, its low incidence and the characteristics of its clinical presentation result in it usually being diagnosed during surgery. Treatment consists in laparoscopic omentenctomy of the affected area. Preoperative diagnosis may allow for watchful waiting, although persistence of symptoms requires readmission to hospital and extension of the definitive treatment.

\section{Resumo}

Introdução: o infarto segmentar idiopático do grande omento é uma causa excepcional de quadro agudo de abdômen no adulto.

Caso clínico: apresenta-se um caso clínico de um adulto jovem operado de urgência por via laparoscópica com diagnóstico de apendicite aguda comprovando-se na exploração um infarto segmentar de grande omento solucionado por uma omentectomia parcial com boa evolução e alta precoce.

Discussão: embora o diagnóstico por técnicas de imagem de infarto do grande omento seja possível, seu diagnóstico habitualmente es intraoperatório devido à sua baja incidência e as características da apresentação clínica. O tratamento é a omentectomia laparoscópica do segmento afetado. Com diagnóstico pré-operatório, pode-se admitir uma conduta expectante, embora a persistência de sintomatologia é causa de re-internação e prolongamento do tratamento definitivo.

\section{Bibliografía}

1. Buell KG, Burke-Smith A, Patel V, Watfah J. Omental infarction: the great impersonator. Cureus 2017; 9(12):e1940.

2. Tonerini M, Calcagni F, Lorenzi S, Scalise P, Grigolini A, Bemi P. Omental infarction and its mimics: imaging features of acute abdominal conditions presenting with fat stranding greater than the decrece of bowel wall thickening. Emerg Radiol 2015; 22(4):431-6.

3. Hussain K, Munir A, Wahla MS, Masood J. Laparoscopic management of primary segmental omental infarction mimicking acute appendicitis. J Coll Physicians Surg Park 2015; 25(Suppl. 2):89-90.

4. Varjavandi V, Lessin M, Kooros K, Fusunyan R, McCauley $\mathbf{R}$, Gilchrist B. Omental infarction: risk factor in children. J Pediatr Surg 2003; 38(2):233-5.

5. McCuster R, Gent R, Goh DW. Diagnosis and management of omental infarction in children: our 10 year experience with ultrasound. J Pediatr Surg 2018; 53(7):1360-4.

6. Gupta R, Farhat W, Ammar H, Azzaza M, Lagha S, Ben M, et al. Idiopathic segmental infarction of the omentum mimicking acute appendicitis?: a case report. Int J Surg Case Rep 2019; 60:66-8.

7. Escaìrcega-Fujigaki $\mathbf{P}$, Hernaìndez Peredo-Rezk G, Hernaìndez- Goìmez $S$, Rodriìguez-Gutiè̀rrez $C$, Martín-Bendimez G, Velasco-Saìnchez R. Infarto segmentarlo idiopático del epiplón mayor, resuelto por cirugía endoscópica. Reporte de caso. Bol Med Hosp Infant Mex 2006; 63(5):322-5.

8. Balthazar EJ, Lefkowitz RA. Left-sided omental infarction with associated omental abscess: CT diagnosis. J Comput Assist Tomogr 1993; 17(3):379-81. 